\title{
Compressed true lumen in the innominate artery: A pitfall of right axillary arterial perfusion in acute aortic dissection
}

\author{
Kazumasa Orihashi, MD, Taijiro Sueda, MD, Kenji Okada, MD, and Shinya Takahashi, MD, Hiroshima, Japan
}

Although axillary arterial perfusion (AX-P) is often preferred to femoral arterial perfusion (FA-P) in acute aortic dissection because it is associated with a lower incidence of malperfusion, it is unlikely to be perfect. ${ }^{1}$ We used near-infrared spectroscopy and orbital Doppler to detect cerebral malperfusion and transesophageal echocardiography (TEE) to clarify the malperfusion mechanism ${ }^{2,3}$ and observed a case of malperfusion after bilateral AX-P, which was relieved after interruption of the right $\mathrm{AX}-\mathrm{P} .{ }^{3}$ We report another case of malperfusion after right AX-P resulting from the narrowing of the innominate artery (IA) lumen.

\section{CLINICAL SUMMARY}

A 46-year-old man had an episode of chest pain 3 days before being transferred to the Hiroshima University Hospital. The patient was in shock and underwent a Bentall operation. Preoperative computed tomography showed a Stanford type A dissection and narrow true lumen of the proximal IA, compressed by a radiopaque false lumen (Figure 1). Intraoperative TEE showed that all arch branches were involved. In the left subclavian and left common carotid arteries, blood flow was detectable in both true and false lumina. The false lumen of the IA was partially echogenic with a mobile intimal flap (Figure 2). The entry was situated in the distal ascending

From the Hiroshima University Hospital, Hiroshima, Japan.

Received for publication Jan 3, 2008; accepted for publication Feb 7, 2008.

Address for reprints: Kazumasa Orihashi, MD, Cardiovascular Surgery, Hiroshima University Hospital, Kasumi 1-2-3, Minami-ku, Hiroshima 734-8551

Japan (E-mail: orichan@hiroshima-u.ac.jp).

J Thorac Cardiovasc Surg 2009; 137:242-3

$0022-5223 / \$ 36.00$

Copyright $(2) 2009$ by The American Association for Thoracic Surgery doi:10.1016/j.jtcvs.2008.02.008 aorta. Right orbital flow was reduced, and right radial artery pressure was lower than the dorsalis pedis artery pressure by $20 \mathrm{~mm} \mathrm{Hg}$. We considered that 1) inflow without outflow inflated the false lumen of the IA, but retrograde AX-P would decompress the true lumen, and that 2) left AX-P or FA-P might lead to false lumen perfusion because reentry could become an entry under retrograde perfusion. After right AX-P, however, dorsalis pedis artery pressure decreased to $30 \mathrm{~mm} \mathrm{Hg}$, and the blood flow in the left subclavian and left common carotid arteries and left orbit became undetectable. TEE revealed that the true lumen of the IA remained narrow.

As left AX-P was added, systemic and left hemisphere perfusion recovered without false lumen perfusion. The near-infrared spectroscopy readings could be maintained within the normal range. Although the narrow true lumen and slight pressure gradient remained, the patient had a favorable postoperative course without complication.

\section{DISCUSSION}

This case suggested another mechanism of malperfusion after AX-P: refractory narrowing of the true lumen in the IA. On the basis of computed tomography and TEE findings, we devised 2 strategies: 1) right AX-P or 2) left AX-P and/or FA-P. However, the initial assessment that the false lumen was flexible was not correct. From a review of the TEE findings, we concluded that the thrombus was echogenic and lacked fluidity. The thrombus might have been formed at the onset of dissection 3 days before. When malperfusion was detected after right AX-P, we had 2 subsequent strategies: FA-P or left AX-P. Although both had a risk of false lumen perfusion because of the

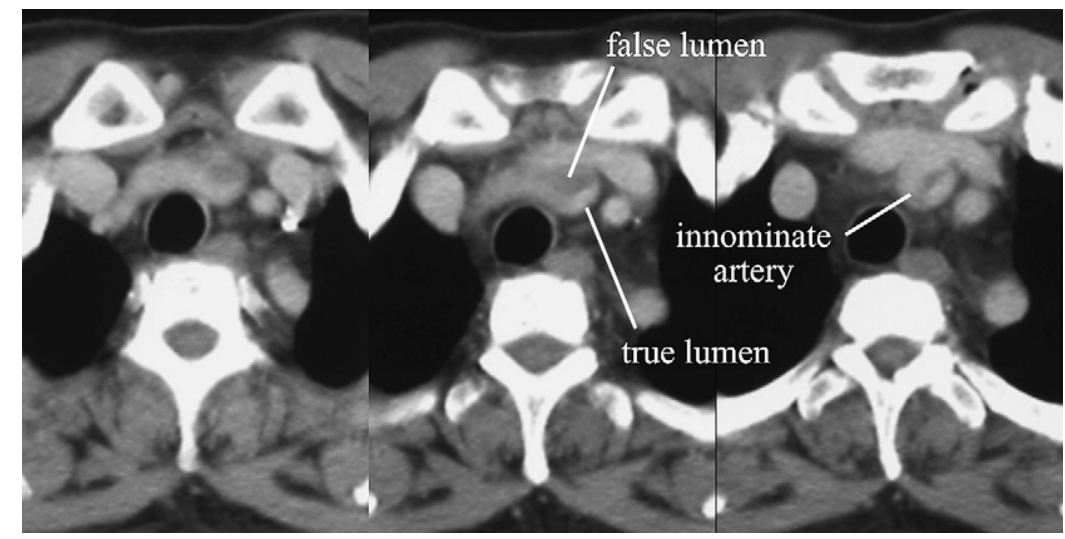

FIGURE 1. Preoperative computed tomography scan demonstrating narrow true lumen with radiopaque false lumen. 


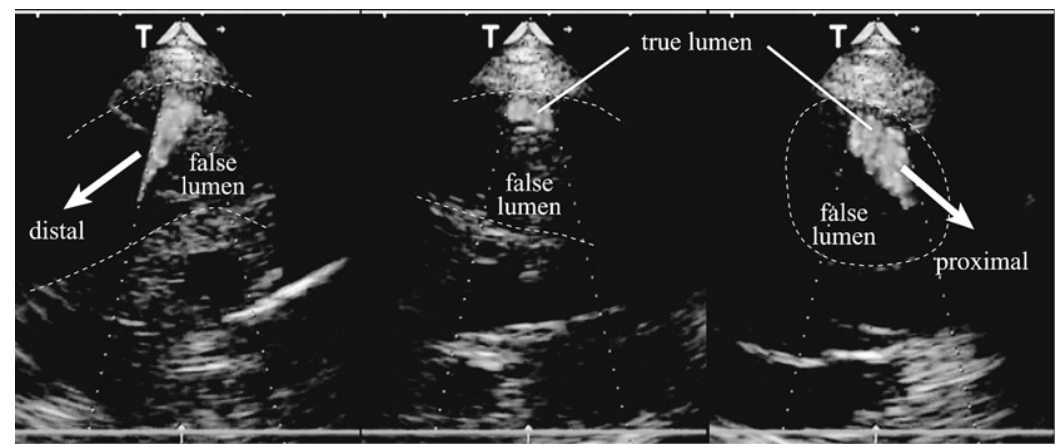

FIGURE 2. Intraoperative transesophageal echocardiogram demonstrating the narrow true lumen of the IA.

presence of reentry, we decided to add left AX-P because the bilateral axillary arteries had been routinely prepared for selective cerebral perfusion at the Hiroshima University Hospital. The addition of FA-P might be another strategy in other institutions.

Because there are unpredictable factors in acute aortic dissection, decisions need to be made stepwise and be based on real-time information at each step. Although our initial assessment was incorrect, TEE and orbital Doppler findings steered the subsequent management toward a good outcome.

Compression of IA can be a mechanism of malperfusion after right AX-P. In acute aortic dissection with many unpre- dictable factors, real-time, on-site information is essential for intraoperative navigation.

\section{References}

1. Pasic M, Schubel J, Bauer M, Yankah C, Kuppe H, Weng YG, et al. Cannulation of the right axillary artery for surgery of acute type A aortic dissection. Eur J Cardiothorac Surg. 2003;24:231-5.

2. Orihashi K, Matsuura Y, Sueda T, Watari M, Okada K, Sugawara Y, et al Aortic arch branches are no longer a blind zone for transesophageal echocardiography: a new eye for aortic surgeons. J Thorac Cardiovasc Surg. 2000; 120:466-72.

3. Orihashi K, Sueda T, Okada K, Imai K. Detection and monitoring of complications associated with femoral or axillary arterial cannulation for surgical repair of aortic dissection. J Cardiothorac Vasc Anesth. 2006;20:20-5.

\title{
Partial left ventricular support implanted through minimal access surgery as a bridge to cardiac transplant
}

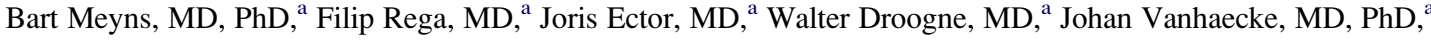 \\ Jan Van Hemelrijck, MD, PhD, ${ }^{\mathrm{a}}$ Bartley Griffith, MD, ${ }^{\mathrm{b}}$ Robert Dowling, MD, ${ }^{\mathrm{c}}$ Mark Zucker, MD, ${ }^{\mathrm{d}}$ and Daniel Burkhoff, MD, PhD, ${ }^{\mathrm{e}}$ \\ Leuven, Belgium, College Park, Md, Louisville, Ky, Newark, NJ and New York, NY
}

The clinical benefits of implantable left ventricular assist devices (LVADs) as a bridge to transplant and for destination therapy have been demonstrated. ${ }^{1-3}$ The low rate of LVAD use is attributed to the invasive nature of the implantation surgery and the relatively high rate of complications. ${ }^{4}$ If

\footnotetext{
From the University Hospital, Leuven, Belgium, ${ }^{\text {a }}$ University of Maryland, College Park, Md, ${ }^{\mathrm{b}}$ University of Louisville, Louisville, Ky, ${ }^{\mathrm{c}}$ Newark Beth Israel Medical Center, Newark, $\mathrm{NJ},{ }^{\mathrm{d}}$ and Columbia University, New York, NY. ${ }^{\mathrm{e}}$

Synergy Micro-Pump (CircuLite, Inc, Hackensack, NJ) was provided by the manufacturer.

Received for publication Dec 30, 2007; accepted for publication Feb 2, 2008.

Address for reprints: Filip Rega, MD, University Hospitals Leuven, Cardiac Surgery, Herestraat 49, 3000 Leuven, Belgium.

J Thorac Cardiovasc Surg 2009;137:243-5

$0022-5223 / \$ 36.00$

Copyright (c) 2009 by The American Association for Thoracic Surgery

doi:10.1016/j.jtcvs.2008.02.010
}

LVADs were used in less critical hemodynamic and clinical states, the flow requirements could be reduced.

The Synergy Micro-Pump (CircuLite, Inc, Hackensack NJ) is approximately the size of a size AA battery, weighs only $25 \mathrm{~g}$, and can pump as much as 2.5 to $3 \mathrm{~L} / \mathrm{min}$. Its small size permits insertion through a right-sided minithoracotomy. The pump is then placed subcutaneously in the pectoral region, similar to a pacemaker. This is the first clinical report describing the Synergy Micro-Pump's implantation procedure, hemodynamic effects, and clinical results.

\section{CLINICAL SUMMARY}

The patient was a 46-year-old man (weight $85 \mathrm{~kg}$, height $184 \mathrm{~cm}$, body surface area $2.01 \mathrm{~m}^{2}$ ) without significant illness in his medical history who had a large anterior myocardial infarction in March 2007. New York Heart Association 